\title{
Multisensor Information Fusion State Estimator for Descriptor and Non Descriptor Systems
}

\author{
Yun $\mathrm{Li}^{1,2}$, Ming Zhao ${ }^{1}$, Gang $\mathrm{Hao}^{2} *$, Junling $\mathrm{Li}^{1}$ and Hao $\mathrm{Jin}^{1}$ \\ 1 School of Computer and Information Engineering \\ Harbin University of Commerce \\ HeiLongJiang, Harbin, 150001, China \\ E-mail: liyunhd@hrbcu.edu.cn \\ $2 *$ Electronic Engineering Institute \\ Heilongjiang University \\ Heilongjiang, Harbin, 150080, China \\ E-mail: haogang@hlju.edu.cn
}

\begin{abstract}
Multisensor distributed information fusion state estimator is presented in this paper. The algorithm deals with descriptor and non descriptor discrete time time-invariant stochastic linear system which is described by state space model. Under the assumption of the observability of the system, the state of the system is a linear combination of input white noise, observation white noise and observation signal. Further non-recursive state estimators algorithm is presented, which can be computed by the white noise estimators and measurement predictor. In order to improve the accuracy of the state estimator, this paper presents information fusion algorithm including matrix weighted, diagonal matrices weighted, scalar weighted and covariance intersection fusion, in the sense of linear minimum variance. The formula of optimal weighting coefficients is given. The algorithm analyzes the relationship between the accuracy and the computation of the four fusion algorithm. A simulation example for non descriptor system with 3 sensors shows its correctness and effectiveness.
\end{abstract}

Keywords: Distributed information fusion, descriptor and non descriptor systems, state estimator

\section{Introduction}

Descriptor system is a kind of power system, and it has a wider application than normal system form. In the literature, it is also named as singular systems, descriptor systems, semi-state systems, generalized state-space systems, differential-algebraic systems, implicit systems and degenerate systems etc.[1]. Descriptor systems occur naturally in robotics, economic, electronic and chemical systems, which are more common than conventional systems to describe the actual systems. The estimation problems for descriptor systems are fundamental to control and synthesis problems for them[2].

With the increasingly requirements of the precision, fault tolerance and robustness of the control systems, the single sensor or measurement equipment is difficult to meet the requirements of the control system [3-5]. Since the 1970s, multi sensor combination and multi sensor information processing problems in the field of robot technology, distributed control system, navigation system, multi target tracking and $\mathrm{C}^{3} \mathrm{I}$ system have been put forward. The multisensor system can obtain more information about the system or information of the target. But the need of a technology is to get to know the target system

*Corresponding author: Gang Hao 
or more precisely from these complementary and redundant information. This technology is information fusion, and is also known as data fusion. Its main principle is to use multiple sensors (homogeneous or heterogeneous) to measure the same object so as to obtain the multi-source information of the object, further integrate the information in order to obtain a better understanding of the observed object. Because of the universality and particularity for the descriptor system in the structure, and it makes the study of state estimation problem of generalized systems is far behind the traditional system ${ }^{[6]}$ ${ }^{\sim 10]}$. The research about multi-sensor information fusion for descriptor system is much less. With the advent of multi-sensor systems, fused estimation problems for multisensor descriptor systems have been received more and more attentions, but they are still in the preliminary stage ${ }^{[11] \sim[12]}$. At present, the problem of state estimation of a descriptor system is mostly based on the observation data of a single sensor. With the popularization of computer application and use of multiple sensors system, we are faced to solve multisensor state estimation problem of descriptor systems ${ }^{[13] \sim[14]}$.

In this paper, distributed information fusion state estimator is weighted by matrix, diagonal matrices, scalars, covariance intersection fusion for descriptor and non descriptor systems. The algorithm is using the modern time series analysis method and white noise estimator under the linear minimum variance sense. The algorithm presented in this paper can handle the state estimation problem of descriptor and non descriptor systems in a unified way, and can also deal with the fused filtering, smoothing and prediction estimation in a unified way. Further unified non-recursive state estimation theories have been proposed in this paper. Its core idea and the key technology include: (1) Using the state space model describing the multi sensor descriptor and non descriptor discrete time time-invariant stochastic linear system; (2) The state of the system is a linear combination of input white noise, observation white noise and observation signal; (3) State estimation is reduced to the problem of computing the white noise estimators and measurement predictor. This paper presents information fusion algorithm including matrix weighted, diagonal matrices weighted, scalar weighted and covariance intersection fusion. The formula of optimal weighting coefficients is given. Compared with the single sensor case, the accuracy of the fused filter is greatly improved. The algorithm analyzes the relationship between the accuracy and the computation of the four fusion algorithm. A simulation example for non descriptor system with 3 sensors shows its correctness and effectiveness.

The main structure of this paper is as follows: Problem formulation is given in Section 2. ARMA innovation model is introduced in Section 3. In Section 4 nonrecursive local optimal state estimator is presented. Distributed information fusion optimal state estimator for descriptor and non descriptor system is given in Section 5. A simulation example with 3 sensors is given in section 6. In Section 7 the conclusions of this paper are given.

\section{Problem Formulation}

Consider the multi sensor descriptor and non descriptor discrete time time-invariant stochastic linear system

$$
\begin{gathered}
\boldsymbol{M x}(t+1)=\Psi \boldsymbol{x}(t)+\Upsilon \boldsymbol{w}(t) \\
\boldsymbol{y}_{j}(t)=\Pi_{j} \boldsymbol{x}(t)+\boldsymbol{v}_{j}(t) \quad j=1, \cdots, k
\end{gathered}
$$

where $t$ is the discrete time and $k$ is the number of the sensor, $k \geq 2$. And $\boldsymbol{x}(t) \in R^{n}$ is the state, the scalar $\boldsymbol{y}_{j}(t) \in R^{m j}$ is the measurement (output) of the $j$ th sensor subsystem, $v_{j}(t) \in R^{m j}$ is the measurement noise of the $j$ th sensor subsystem, $\boldsymbol{\omega}(t) \in R^{r}$ is the input noise, $\boldsymbol{M}, \Psi, \Upsilon, \Pi_{j}$ is the known constant matrix. 
Assumption $1 \boldsymbol{M}$ is singular matrix ( $\operatorname{det} \boldsymbol{M}=0$ ) or $\boldsymbol{M}=\boldsymbol{I}_{n}$ (unit matrix).

Assumption 2 The system is regular.

Assumption $3 \boldsymbol{w}(t) \in R^{r}$ and $\boldsymbol{v}_{i}(t) \in R^{m i}, i=1, \cdots, L$ are independence white noises with zero mean and covariance are $Q_{w}$ and $Q_{v i}$ individually.

$$
\mathrm{E}\left\{\left[\begin{array}{c}
\boldsymbol{w}(t) \\
\boldsymbol{v}_{j}(t)
\end{array}\right]\left[\begin{array}{ll}
\boldsymbol{w}^{\mathrm{T}}(k) & \boldsymbol{v}_{i}^{\mathrm{T}}(k)
\end{array}\right]\right\}=\left[\begin{array}{cc}
Q_{w} & S_{i} \\
S_{j}^{\mathrm{T}} & Q_{v_{j i}}
\end{array}\right] \delta_{t k}
$$

where $\mathrm{E}$ is the mathematical expectation, $\delta_{t t}=1, \delta_{t k}=0(t \neq k)$.

Assumption 4 The system is completely observable, that is, for the arbitrary complex $z$ have

$$
\operatorname{rank}\left[\begin{array}{c}
z \boldsymbol{M}-\Psi \\
\Pi_{j}
\end{array}\right]=n, \operatorname{rank}\left[\begin{array}{c}
\boldsymbol{M} \\
\Pi_{j}
\end{array}\right]=n
$$

The following the observable matrix is a column full rank that is equivalent to the Assumption 4.

$$
\Omega_{j}=\left[\begin{array}{ccccc}
-\Psi & M & \cdots & 0 & 0 \\
\vdots & \vdots & & \vdots & \vdots \\
0 & 0 & & -\Psi & M \\
\Pi_{j} & 0 & \cdots & 0 & 0 \\
\vdots & \vdots & & \vdots & \vdots \\
0 & 0 & \cdots & 0 & \Pi_{j}
\end{array}\right], \Theta_{j}=\left[\begin{array}{ccccc}
M & -\Psi & \cdots & 0 & 0 \\
\vdots & \vdots & & \vdots & \vdots \\
0 & 0 & & M & -\Psi \\
\Pi_{j} & 0 & \cdots & 0 & 0 \\
\vdots & \vdots & & \vdots & \vdots \\
0 & 0 & \cdots & 0 & \Pi_{j}
\end{array}\right]
$$

Where the order of $\Omega_{j}$ and $\Theta_{j}$ is $[(\beta-1) n+\beta m] \times \beta n$, and $\beta$ is named as the observability index.

State estimation problem for descriptor and non descriptor system is based on the measurement $\left(\boldsymbol{y}_{i}(t+N), \boldsymbol{y}_{i}(t+N-1), \cdots\right)$, to obtain non-recursive state estimators $\hat{\boldsymbol{x}}_{j}(t \mid t+N), j=1,2 \cdots K$. For $N=0, N>0$ or $N<0$, we named it as state filtering, smoothing or predictor. Further distributed optimal information fusion non-recursive state estimation $\hat{\boldsymbol{x}}_{0}(t \mid t+N)$ is obtained, it consists of weighted local state estimators.

\section{ARMA Innovation Model}

Lemma 1 For the system (1) and (2) under the Assumption 1-2, and having the left prime decomposition ${ }^{[15]}$ :

$$
\Pi_{j}\left(\boldsymbol{M}-q^{-1} \Psi\right)^{-1} \Upsilon q^{-1}=\boldsymbol{A}^{-1}\left(q^{-1}\right) \boldsymbol{B}^{-1}\left(q^{-1}\right) q^{\tau}
$$

where $\boldsymbol{A}\left(q^{-1}\right)$ and $\boldsymbol{B}^{-1}\left(q^{-1}\right)$ is polynomial matrix of the unit delay operator $q^{-1}$, and it has the type as $X\left(q^{-1}\right)=X_{0}+X_{1} q^{-1}+\cdots+X_{n_{x}} q^{-n_{x}} . X_{i}$ is the coefficient matrix, and $n_{x}$ is the order, and $q$ is the unit forward operator. $\boldsymbol{A}_{0}=I_{m}$, and $B_{0} \neq 0$. Where $\tau$ is the integer, $\tau=0, \tau>0$ or $\tau<0$.

From (1) and (2) having

$$
y_{i}(t)=\Pi_{j}\left(\mathbf{M}-q^{-1} \Psi\right)^{-1} \Upsilon q^{-1} w(t)+v(t)
$$

Substitutting (6) into (7), having

$$
A^{(j)}\left(q^{-1}\right) y_{j}(t)=B^{(j)}\left(q^{-1}\right) q^{\tau} w(t)+A^{(j)}\left(q^{-1}\right) v_{j}(t)
$$

Because of left coprime factorization, and having the ARMA innovation model

$$
A^{(j)}\left(q^{-1}\right) y_{j}(t)=D^{(j)}\left(q^{-1}\right) \varepsilon_{j}(t)
$$


where $D^{(j)}\left(q^{-1}\right)$ is stable, $D_{0}^{(j)}=I_{m}$, and innovation $\varepsilon_{j}(t)$ is white noises with zero mean and covariance are $Q_{\varepsilon j}$, and having

$$
D^{(j)}\left(q^{-1}\right) \varepsilon_{j}(t)=B^{(j)}\left(q^{-1}\right) w(t)+A^{(j)}\left(q^{-1}\right) v_{j}(t)
$$

$D^{(j)}\left(q^{-1}\right)$ and $Q_{\varepsilon j}$ can be computed by Gevers-Wouters ${ }^{[15]}$.

\section{Non-recursive Local Optimal State Estimator}

Lemma 2 For the system (1) and (2) under the Assumption 1-3, the following formula is established ${ }^{[15]}$ :

$$
\begin{aligned}
\mathrm{E}\left[w(t) \varepsilon_{j}^{\mathrm{T}}(i)\right] & =Q_{w} F_{i-t+\max (\tau, 0)}^{\mathrm{T}(j)}+S_{j} G_{i-t+\max (\tau, 0)}^{\mathrm{T}(j)} \\
\mathrm{E}\left[v_{j}(t) \varepsilon_{j}^{\mathrm{T}}(i)\right] & =Q_{v i} G_{i-t+\max (\tau, 0)}^{\mathrm{T}(j)}+S_{j}^{\mathrm{T}} F_{i-t+\max (\tau, 0)}^{\mathrm{T}(j)}
\end{aligned}
$$

The coefficient $F_{i}^{(j)}$ and $G_{i}^{(j)}$ can be recursive calculated as

$$
\begin{aligned}
& F_{i}^{(j)}=-D_{1}^{(j)} F_{i-1}^{(j)}-\cdots-D_{n_{d}}^{(j)} F_{j-n_{d}}^{(j)}+\bar{B}_{i}^{(j)} \\
& G_{i}^{(j)}=-D_{1}^{(j)} G_{i-1}^{(j)}-\cdots-D_{n_{d}}^{(j)} G_{j-n_{d}}^{(j)}+\bar{A}_{i}^{(j)}
\end{aligned}
$$

where letting $F_{i}^{(j)}=0(i<0), \bar{B}_{i}^{(j)}=0\left(i>n_{\bar{b}}\right), G_{i}^{(j)}=0(i<0), \bar{A}_{i}^{(j)}=0\left(i>n_{\bar{a}}\right)$.

$$
\bar{B}^{(j)}\left(q^{-1}\right)=B^{(j)}\left(q^{-1}\right) q^{\min (\tau, 0)}, \bar{A}^{(j)}\left(q^{-1}\right)=A^{(j)}\left(q^{-1}\right) q^{\min t, 0}
$$

Lemma 3 For the system (1) and (2) under the Assumption 1-3, the asymptotically stability local optimal white estimator is as follows ${ }^{[15]}$

When $N \geq-\max (\tau, 0)$, we have

$$
\begin{aligned}
& \hat{w}(t \mid t+N)=\sum_{i=-\max (\tau \vee 0)}^{N}\left[Q_{w} F_{i+\max (\tau \vee \mathrm{v} 0)}^{(j) \mathrm{T}}+S_{j} G_{i+\max (\tau \mathrm{v} 0)}^{(j) \mathrm{T}}\right] Q_{\varepsilon}^{(j)-1} \varepsilon_{j}(t+i) \\
& \hat{v}_{j}(t \mid t+N)=\sum_{i=-\max (\tau \vee 0)}^{N}\left[Q_{v j} G_{i+\max (\tau \mathrm{v} 0)}^{(j \mathrm{~T})}+S_{j}^{\mathrm{T}} F_{i+\max (\tau \mathrm{v} 0)}^{(j) \mathrm{T}}\right] Q_{\varepsilon}^{(j)-1} \varepsilon_{j}(t+i)
\end{aligned}
$$

When $N<-\max (\tau, 0)$, we have

$$
\hat{w}(t \mid t+N)=0, \hat{v}(t \mid t+N)=0
$$

Lemma 4 The measurement $y_{j}(t+i)$ has the recursive predictor ${ }^{[15]}$

$$
A^{(j)}\left(\tilde{q}^{-1}\right) \hat{y}_{j}(t+N \mid t)=D^{(j)}\left(q^{-1}\right) \varepsilon_{j}(t+j), \quad N=1, \cdots, i
$$

where letting $\varepsilon_{j}(t+j)=0(j>0)$, and $\hat{y}_{j}(i \mid N)=y(i)(i \leq j)$.

$$
A^{(j)}\left(\tilde{q}^{-1}\right) \hat{y}_{j}(t+N \mid t)=\hat{y}_{j}(t+N \mid t)+A_{1}^{(j)} \hat{y}_{j}(t+N-1 \mid t)+\cdots+A_{n_{a}}^{(j)} \hat{y}_{j}\left(t+N-n_{a} \mid t\right)
$$

And innovation $\varepsilon_{i}(t)$ can be calculated by

$$
\varepsilon_{j}(t)=A^{(j)}\left(q^{-1}\right) y(t)-D_{1}^{(j)} \varepsilon_{j}(t-1)-\cdots-D_{n_{d}}^{(j)} \varepsilon_{j}\left(t-n_{d}\right), t=n_{d}, n_{d}+1, \cdots
$$

Theorem 1 For the system (1) and (2) under the Assumption 1-4, asymptotically stability state estimator is as follows

$$
\hat{x}(t \mid t+N)=\sum_{i=0}^{\beta-2} \Omega_{i}^{(j 1)} \Pi \hat{w}(t+i \mid t+N)+\sum_{i=0}^{\beta-1} \Omega_{i}^{(j 2)}\left[\hat{y}_{j}(t+i \mid t+N)-\hat{v}_{j}(t+i \mid t+N)\right]
$$

Defining $\Omega_{i}^{(j)}$ as 


$$
\Omega_{j}^{+}=\left(\Omega_{j}^{\mathrm{T}} \Omega_{j}\right)^{-1} \Omega_{j}^{\mathrm{T}}=\left[\begin{array}{cccccc}
\Omega_{0}^{(j 1)} & \cdots & \Omega_{\beta-2}^{(j 1)} & \Omega_{0}^{(j 2)} & \cdots & \Omega_{\beta-1}^{(j 2)} \\
\vdots & & \vdots & \vdots & & \vdots \\
\vdots & & \vdots & \vdots & & \vdots
\end{array}\right]
$$

where $\Omega_{j}$ is defined by (5).

Proof: Form (1) and (2) have the relationship

$$
\Omega_{j}\left[\begin{array}{c}
x(t) \\
x(t+1) \\
\vdots \\
\vdots \\
\vdots \\
x(t+\beta-1)
\end{array}\right]=\left[\begin{array}{c}
\Upsilon w(t) \\
\vdots \\
\Upsilon w(t+\beta-2) \\
y(t)-v(t) \\
\vdots \\
y(t+\beta-1)-v(t+\beta-1)
\end{array}\right]
$$

By (23) and (24) yield

$$
x(t)=\sum_{i=0}^{\beta-2} \Omega_{i}^{(j 1)} \Pi w(t+i)+\sum_{i=0}^{\beta-1} \Omega_{i}^{(j 2)}\left[y_{j}(t+i)-v_{j}(t+i)\right]
$$

Taking on (25) projective operation, and (22) is obtained. Due to white noise estimators and measurement predictor is asymptotically stable and asymptotically optimal about innovation initial value. So (22) also has the same properties. The proof is completed.

Theorem 2 For the system (1) and (2) under the Assumption 1-4, estimation error $\tilde{x}(t \mid t+N)=x(t)-\hat{x}(t \mid t+N)$ has the following expressions

$$
\tilde{x}_{j}(t \mid t+N)=\sum_{i=0}^{\beta_{j}-2} \Omega_{i}^{(j) w} w_{j}(t+i)-\sum_{i=0}^{\beta_{j}-1} \Omega_{i}^{(j) v} \boldsymbol{v}_{j}(t+i)+\sum_{i=0}^{n_{0}} \Omega_{i}^{(j) c} \varepsilon_{j}(t+i), \quad N \geq 0
$$

Where defining $n_{0}=\max \left(\beta_{j}-1, N\right), \Omega_{j}^{(i) c}$ is obtained by the merger of similar coefficient matrix.

$$
\tilde{\boldsymbol{x}}_{j}(t \mid t+N)=\sum_{i=0}^{\beta_{j}-2} \Omega_{i}^{(j) w} \boldsymbol{w}_{j}(t+i)-\sum_{i=0}^{\beta_{j}-1} \Omega_{i}^{(j) v} \boldsymbol{v}_{j}(t+i)+\sum_{i=0}^{n_{1}} \Omega_{i}^{(j) y} \boldsymbol{\varepsilon}_{j}(t+N+1+i), \quad N<0
$$

Where defining $n_{1}=\left(\beta_{i}-2-N\right), \Omega_{i}^{(j) y}$ is obtained by the merger of similar coefficient matrix. (26) and (27) can be expressed as a unified form

$$
\begin{gathered}
\tilde{\boldsymbol{x}}_{j}(t \mid t+N)=\sum_{i=0}^{n_{0}}\left[\Omega_{i}^{(j) w} \boldsymbol{w}_{j}(t+i)-\Omega_{i}^{(j) v} \boldsymbol{v}_{j}(t+i)+\Omega_{i}^{(j) \mathrm{c}} \boldsymbol{\varepsilon}_{j}(t+i)\right], \quad N \geq 0 \\
\tilde{\boldsymbol{x}}_{i}(t \mid t+N)=\sum_{j=0}^{n_{1}}\left[\Omega_{j}^{(i) w} \boldsymbol{w}_{i}(t+j)-\Omega_{j}^{(i) v} \boldsymbol{v}_{i}(t+j)+\Omega_{j}^{(i) y} \boldsymbol{\varepsilon}_{i}(t+N+1+j)\right], \quad N<0
\end{gathered}
$$

And letting $\Omega_{i}^{(j) w}=0\left(i>\beta_{j}-2\right), \Omega_{i}^{(j) v}=0\left(i>\beta_{j}-1\right)$.

Proof: (25) minus (22), then mergers similar items, (28)and (29) are obtained. The proof is completed.

Theorem 3 For the system (1) and (2) under the Assumption 1-4, estimation error covariance $P_{j}(N)=\mathrm{E}\left[\tilde{\boldsymbol{x}}_{j}(t \mid t+N) \tilde{\boldsymbol{x}}_{j}^{\mathrm{T}}(t \mid t+N)\right]$ is given as

$$
P_{j}(N)=\sum_{r=0}^{n_{0}} \sum_{s=0}^{n_{0}}\left[\begin{array}{lll}
\Omega_{r}^{(j) w} & -\Omega_{r}^{(j) v} & \Omega_{r}^{(j) c}
\end{array}\right]\left[\begin{array}{ccc}
Q_{\bar{w}_{j j}} \delta_{r s} & \bar{S}_{j} \delta_{r s} & \Lambda_{s-r}^{(j) \bar{w}_{j}} \\
\bar{S}_{j}^{\mathrm{T}} \delta_{r s} & Q_{v_{j}} \delta_{r s} & \Lambda_{s-r}^{(j) v_{j}} \\
\Lambda_{r-s}^{(j) \bar{w}_{j} \mathrm{~T}} & \Lambda_{r-s}^{(j) v_{j} \mathrm{~T}} & Q_{\varepsilon j} \delta_{r s}
\end{array}\right]\left[\begin{array}{c}
\Omega_{s}^{(j) w \mathrm{~T}} \\
-\Omega_{s}^{(j) v \mathrm{~T}} \\
\Omega_{s}^{(j) c \mathrm{~T}}
\end{array}\right], N \geq 0
$$




$$
P_{j}(N)=\sum_{r=0}^{n_{1}} \sum_{s=0}^{n_{1}}\left[\begin{array}{lll}
\Omega_{r}^{(j) w} & -\Omega_{r}^{(j) v} & \Omega_{r}^{(j) y}
\end{array}\right]\left[\begin{array}{ccc}
Q_{\bar{w}_{i j}} \delta_{r s} & \bar{S}_{j} \delta_{r s} & \Lambda_{N+1+s-r}^{(j) \bar{w}_{j}} \\
\bar{S}_{j}^{\mathrm{T}} \delta_{r s} & Q_{v_{j}} \delta_{r s} & \Lambda_{N+1+s-r}^{(j) v_{j}} \\
\Lambda_{N+1+r-s}^{(j) \bar{m}_{j} \mathrm{~T}} & \Lambda_{N+1+r-s}^{(j) v_{j} \mathrm{~T}} & Q_{\varepsilon j} \delta_{r s}
\end{array}\right]\left[\begin{array}{c}
\Omega_{s}^{(j) w \mathrm{~T}} \\
-\Omega_{s}^{(j) v \mathrm{~T}} \\
\Omega_{s}^{(j) y \mathrm{~T}}
\end{array}\right], \quad N<0
$$

the cross covariance $P_{i j}(N)=\mathrm{E}\left[\tilde{\boldsymbol{\alpha}}_{i}(t \mid t+N) \tilde{\boldsymbol{\alpha}}_{j}^{\mathrm{T}}(t \mid t+N)\right]$ between any two local sensor is computed by

$$
\begin{aligned}
& P_{i j}(N)=\sum_{r=0}^{n_{0}} \sum_{s=0}^{n_{0}}\left[\begin{array}{lll}
\Omega_{r}^{(i) w} & -\Omega_{r}^{(i) v} & \Omega_{r}^{(i) c}
\end{array}\right]\left[\begin{array}{ccc}
Q_{\bar{w}_{i j}} \delta_{r s} & \bar{S}_{j} \delta_{r s} & \Lambda_{s-r}^{(j) \overline{w_{i}}} \\
\bar{S}_{i}^{\mathrm{T}} \delta_{r s} & Q_{v_{i j}} \delta_{r s} & \sum_{i j}^{v \varepsilon}(r, s) \\
\Lambda_{r-s}^{(i) \bar{w}_{i} \mathrm{~T}} & \sum_{j i}^{v \in \mathrm{T}}(r, s) & \sum_{i j}^{\varepsilon \varepsilon}(r, s)
\end{array}\right]\left[\begin{array}{c}
\Omega_{s}^{(j) w \mathrm{~T}} \\
-\Omega_{s}^{(j) v \mathrm{~T}} \\
\Omega_{s}^{(j) \mathrm{T}}
\end{array}\right], \quad N \geq 0 \\
& P_{i j}(N)=\sum_{r=0}^{n_{1}} \sum_{s=0}^{n_{1}}\left[\begin{array}{lll}
\Omega_{r}^{(i) w} & -\Omega_{r}^{(i) v} & \Omega_{r}^{(i) y}
\end{array}\right]\left[\begin{array}{ccc}
Q_{\bar{w}_{j}} \delta_{r s} & \bar{S}_{j} \delta_{r s} & \Lambda_{N+1+r-s}^{(j) \bar{w}_{i}} \\
\bar{S}_{i}^{\mathrm{T}} \delta_{r_{s \mathrm{~s}}} & Q_{v_{j}} \delta_{r s} & \sum_{i j}^{v E}(r, N+1+s) \\
\Lambda_{N+1+r-s}^{(i) \bar{w}_{i} \mathrm{~T}} & \sum_{j i}^{v \varepsilon}(r, N+1+r) & \sum_{i j}^{\varepsilon \varepsilon}(N+1+r, N+1+s)
\end{array}\right] \times\left[\begin{array}{c}
\Omega_{s}^{(j) w \mathrm{~T}} \\
-\Omega_{s}^{(j) v \mathrm{~T}} \\
\Omega_{s}^{(j) y \mathrm{~T}}
\end{array}\right], N<0 \\
& \sum_{j i}^{v \varepsilon}(r, s)=\bar{S}_{j}^{\mathrm{T}} F_{s-r}^{(i) \mathrm{T}}+Q_{v_{i j}^{\mathrm{T}}}^{\mathrm{T}} G_{s-r}^{(i) \mathrm{T}} \\
& \sum_{i j}^{v \varepsilon}(r, s)=\bar{S}_{i}^{\mathrm{T}} F_{s-r}^{(j) \mathrm{T}}+Q_{v_{i j}} G_{s-r}^{(j) \mathrm{T}} \\
& \sum_{i j}^{\varepsilon \varepsilon}(r, s)=\sum_{k=0}^{\infty} F_{k}^{(i)} Q_{\overline{w_{i j}}} F_{k+s-r}^{(j) \mathrm{T}}+\sum_{k=0}^{\infty} F_{k}^{(i)} \bar{S}_{j} G_{k+s-r}^{(j) \mathrm{T}}+\sum_{k=0}^{\infty} G_{k}^{(i)} S_{i}^{\mathrm{T}} F_{k+s-r}^{(j) \mathrm{T}}+\sum_{k=0}^{\infty} G_{k}^{(i)} Q_{v_{i j}} G_{k+s-r}^{(j) \mathrm{T}}
\end{aligned}
$$

Proof: (28) and (29)have the simplified expression

$$
\begin{gathered}
\tilde{\alpha}_{j}(t \mid t+N)=\sum_{r=0}^{n_{0}}\left[\begin{array}{lll}
\Omega_{r}^{(j) w} & -\Omega_{r}^{(j) v} & \Omega_{r}^{(j) c}
\end{array}\right]\left[\begin{array}{c}
w(t+r) \\
v_{j}(t+r) \\
\varepsilon_{j}(t+r)
\end{array}\right], N \geq 0 \\
\tilde{\alpha}_{j}(t \mid t+N)=\sum_{r=0}^{n_{1}}\left[\begin{array}{lll}
\Omega_{r}^{(j) w} & -\Omega_{r}^{(j) v} & \Omega_{r}^{(j) y}
\end{array}\right]\left[\begin{array}{c}
w(t+r) \\
v_{j}(t+r) \\
\varepsilon_{j}(t+N+1+r)
\end{array}\right], N<0
\end{gathered}
$$

Thus by (3), (16) and (17) with the Assumption 1, (30) (36) is obtained. The proof is completed.

\section{Distributed Information Fusion Optimal State Estimator}

Lemma 5 For the system (1) and (2) under the Assumption 1-4, and the optimal fused state filtering $\hat{x}_{0}(t \mid t+N)$ weighted by matrices in the linear minimum variance sense is given as ${ }^{[15]}$

$$
\hat{\boldsymbol{x}}_{0}(t \mid t+N)=\sum_{j=1}^{k} A_{j}(N) \hat{\boldsymbol{x}}_{j}(t \mid t+N)
$$

Under the linear minimum variance optimal information fusion criterion which minimize the performance index, the optimal weighting coefficients $A_{j}(N)$, $j=1,2, \cdots, k$ are given by

$$
\left[A_{1}(N), \cdots, A_{l}(N)\right]=\left(e^{T} P^{-1}(N) e\right)^{-1} e^{T} P^{-1}(N)
$$

where 


$$
P(N)=\left[\begin{array}{cccc}
P_{11}(N) & P_{12}(N) & \cdots & P_{1 l}(N) \\
P_{21}(N) & P_{22}(N) & \cdots & P_{2 l}(N) \\
\cdots & \cdots & \cdots & \cdots \\
P_{l 1}(N) & P_{l 2}(N) & \cdots & P_{l l}(N)
\end{array}\right], \quad e=\left[\begin{array}{c}
I_{m} \\
I_{m} \\
\vdots \\
I_{m}
\end{array}\right], \quad P_{j j}(N)=P_{j}(N)(j=1,2, \cdots, k)
$$

Where $P_{j}(N)$ and $P_{j i}(N)$ can be calculated by Theorem 3 .

The optimal fused variance matrix is given as

$$
P_{0}(N)=\left(e^{T} P^{-1}(N) e\right)^{-1}
$$

and

$$
\operatorname{tr} \boldsymbol{P}_{0} \leq \operatorname{tr} \boldsymbol{P}_{j}, j=1,2, \cdots, k
$$

Lemma 6 For the system (1) and (2) under the same condition, and the optimal fused state filtering $\hat{\boldsymbol{x}}_{0}(t \mid t+N)$ weighted by scalars in the linear minimum variance sense is given as ${ }^{[15]}$

$$
\hat{\boldsymbol{x}}_{0}(t \mid t+N)=\sum_{i=1}^{l} \alpha_{i}(N) \hat{\boldsymbol{x}}_{i}(t \mid t+N)
$$

Under the linear minimum variance optimal information fusion criterion, the optimal weighting coefficients $\alpha_{i}(N), j=1,2, \cdots, k$ are given by

$$
\left[\alpha_{1}(N), \cdots, \alpha_{l}(N)\right]=\frac{e^{T} \bar{P}^{-1}(N)}{\left(e^{T} \bar{P}^{-1}(N) e\right)}
$$

where $\bar{P}=\left(\operatorname{tr} P_{i j}(N)\right)_{l \times l}, i, j=1,2, \cdots, l$ is a $l \times l$ matrix, $P_{i j}(N)$ can be calculated by (32) (36), and $e^{\mathrm{T}}=[1, \cdots, 1]$ is a $l \times 1$ row vector.

The optimal fused variance matrix is given as

$$
P_{0}(N)=\sum_{i, j=1}^{l} \alpha_{i} \alpha_{j} P_{i j}(N)
$$

and

$$
\operatorname{tr} P_{0}(N) \leq \operatorname{tr} P_{i}(N), i=1,2, \cdots, l
$$

Lemma 7 For the system (1) and (2) under the same condition, and the optimal fused state filtering $\hat{\boldsymbol{x}}_{0 i}(t \mid t+N)$ weighted by diagonal matrices in the linear minimum variance sense is given as ${ }^{[15]}$

$$
\hat{\boldsymbol{x}}_{0 i}(t \mid t+N)=\alpha_{1 i} \hat{\boldsymbol{x}}_{1 i}(t \mid t+N)+\alpha_{2 i} \hat{\boldsymbol{x}}_{2 i}(t \mid t+N)+\cdots+\alpha_{l i} \hat{\boldsymbol{x}}_{l i}(t \mid t+N), i=1, \cdots, n
$$

The weighted coefficients $\boldsymbol{A}_{j}=\operatorname{diag}\left(a_{j k}\right), k=1, \cdots, n$, while $a_{j k}$ are given by

$$
\left[\alpha_{1}(N), \cdots, \alpha_{l}(N)\right]=\frac{e^{T}\left(P^{i i}(N)\right)^{-1}}{\left(e^{T}\left(P^{i i}(N)\right)^{-1} e\right)}, i=1, \cdots, l
$$

where

$$
P^{i i}(N)=\left[\begin{array}{ccc}
P_{11}^{(i i)}(N) & \cdots & P_{1 l}^{(i i)}(N) \\
\vdots & \ddots & \vdots \\
P_{l 1}^{(i i)}(N) & \cdots & P_{l l}^{(i i)}(N)
\end{array}\right], i=1, \cdots, l
$$


where $e=\left[\begin{array}{lll}1 & \cdots & 1\end{array}\right]^{\mathrm{T}}$ is a $L \times 1$ row vector, and $L \times L$ matrices is defined as $P^{j j}(t \mid t)=\left(P_{l k}^{i j}(t \mid t)\right), \quad l, k=1,2, \cdots, L, P_{k j}^{(i i)}(N)$ is the $j$ th row and $j$ th column diagonal element of $P_{k j}(N) . P_{i j}(N)$ is computed by Theorem 3.

The optimal fused variance matrix of each component is given as

$$
P_{0 i}(N)=\left[e^{T}\left(P^{i i}(N)\right)^{-1} e\right]^{-1}
$$

and

$$
\operatorname{tr} P_{0 i}(N) \leq \operatorname{tr} P_{k k}^{i i}, i=1,2, \cdots, n, \quad k=1,2, \cdots, l
$$

The trace of minimum estimation error covariance matrix is

$$
\operatorname{tr} P_{0}(N)=\operatorname{tr} P_{01}(N)+\cdots+\operatorname{tr} P_{0 n}(N)
$$

and

$$
\operatorname{tr} P_{0}(N) \leq \operatorname{tr} P_{i}(N), i=1,2, \cdots, l
$$

Lemma 8 For the system (1) and (2), under the same conditions, when the variance of $P_{1}$ and $P_{2}$ are known, but the cross covariance $P_{12}$ is unknown, using the covariance intersection (CI) fusion method, this paper proposes a suboptimal fusion Kalman estimators is as follows:

$$
\hat{\boldsymbol{x}}_{C I}(t \mid t+N)=\boldsymbol{P}_{C l}\left[\omega \boldsymbol{P}_{1}^{-1} \hat{\boldsymbol{x}}_{1}(t \mid t+N)+(1-\omega) \boldsymbol{P}_{2}^{-1} \hat{\boldsymbol{x}}_{2}(t \mid t+N)\right]
$$

where $\omega \in[0,1]$ and minimizes the performance index

$$
J=\min _{\omega} \operatorname{tr} \boldsymbol{P}_{C I}
$$

and $\boldsymbol{P}_{C I}$ is defined as

$$
\boldsymbol{P}_{C I}=\left[\omega \boldsymbol{P}_{1}^{-1}+(1-\omega) \boldsymbol{P}_{2}^{-1}\right]^{-1}
$$

For the non-linear optimization problems (57), the optimal weights $\omega$ can be obtained by 0.618 method or the Fabonacci method ${ }^{[16]}$.

\section{Simulation Example}

Consider the multi sensor descriptor and non descriptor discrete time time-invariant stochastic linear system

$$
\begin{gathered}
\boldsymbol{M x}(t+1)=\Psi \boldsymbol{x}(t)+\Upsilon \boldsymbol{w}(t) \\
\boldsymbol{y}_{j}(t)=\Pi_{j} \boldsymbol{x}(t)+\boldsymbol{\xi}_{j}(t) \quad j=1, \cdots, K \\
\boldsymbol{\xi}_{j}(t+1)=F(t) \times \boldsymbol{\xi}_{j}(t)+\boldsymbol{\zeta}_{j}(t)
\end{gathered}
$$

where $\mathrm{T}=1$ is the sampled period, $\Psi=\left[\begin{array}{ccc}1 & \mathrm{~T} & 0.5 \times \mathrm{T}^{2} \\ 0 & 1 & \mathrm{~T} \\ 0 & 0 & 1\end{array}\right], \quad \Upsilon=\left[\begin{array}{lll}\mathrm{T}^{3} / 6 & \mathrm{~T}^{2} / 2 & \mathrm{~T}\end{array}\right]^{\mathrm{T}}$, $H_{1}=H_{2}=H_{3}=\left[\begin{array}{lll}1 & 0 & 0\end{array}\right], F_{1}=0.5, F_{2}=0.3$. And $\xi_{j}(t)$ is white noises with zero mean, $\sigma_{\xi 1}^{2}=0.1, \sigma_{\xi_{2}}^{2}=0.5$. And $w(t)$ is white noises with zero mean and its covariance is $\sigma_{w}^{2}=0.8$. 
State estimation problem is based on the measurement $\left(\boldsymbol{y}_{i}(t+N), \boldsymbol{y}_{i}(t+N-1), \cdots\right)$, to obtain the linear minimum variance fusion state $\hat{\boldsymbol{x}}_{0}(t \mid t)$.

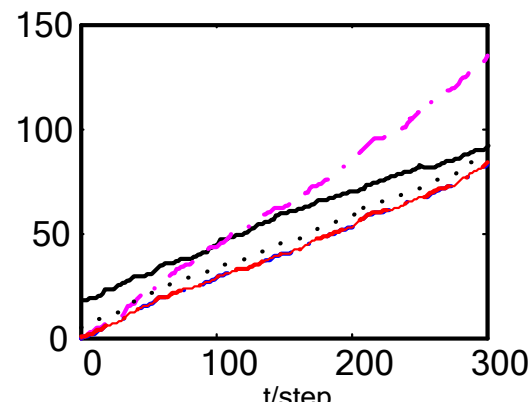

Fig. 1 The curves of the sum of absolute erro curve for local and fusion filters of the position

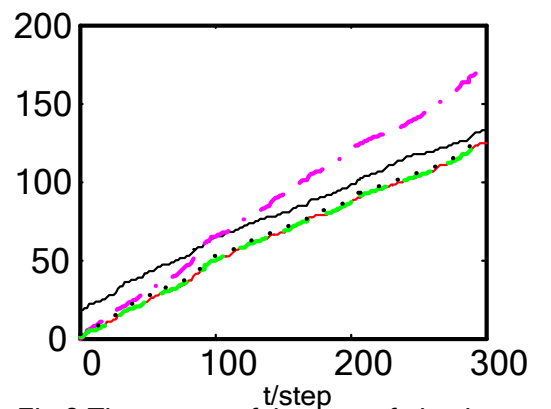

Fig. 3 The curves of the sum of absolute error curve for local and fusion filters of the acceleration

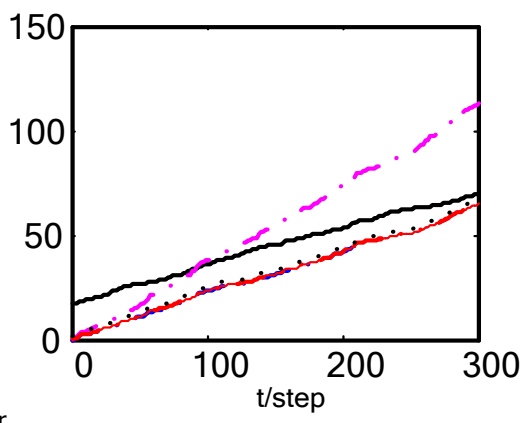

Fig.2 The curves of the sum of absolute error curve for local and fusion filters of the velocity

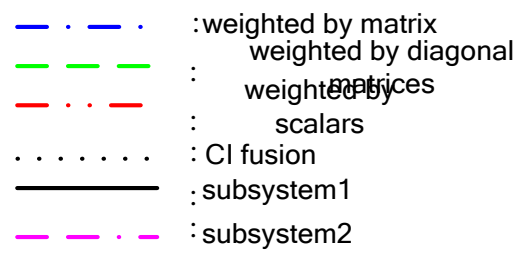

The simulation results are shown in Fig.1-Fig.3. Fig.1-Fig.3 are the absolute error curves for the local and fusion state smooth weighted by matrix, diagonal matrices, scalars, covariance intersection fusion. In the figure, the accuracy of the fusion signal estimator is higher than any of the single sensor. Simulation results show no significant difference between the three kinds of distributed fusion algorithm (matrix, diagonal matrices, scalars), while the accuracy of covariance intersection fusion is lower than the other three fusion algorithm. But the covariance intersection fusion state estimator has the minimal computational burden because of avoiding computing the cross covariance matrix, and it is suitable for real-time applications.

\section{Conclusions}

Under the linear minimum variance optimal information fusion criterion, based on state-space model, the white noise estimator and the observation predictor are applied in this algorithm. Modern time series analysis method and GeversWouters(G-W) algorithm are also used in this paper, and the multi sensor optimal information fusion state estimator for descriptor and non descriptor systems is presented. The algorithm presented in this paper has many advantages:

(1) Non recursive state estimators can not only deal with the estimation problem of descriptor and non descriptor systems in a unified way, and can also handle the filtering, smoothing and prediction problems in a unified way.

(2) Sufficient conditions of the non-recursive steady state estimators are the objectivity.

(3) Non-recursive state estimators can be applied to non-square descriptor systems. 
(4) Distributed information fusion rule, which adopted in this paper, is weighted by matrix, diagonal matrices, scalars, covariance intersection fusion. The estimation accuracy for the system is greatly improved compared with the single local sensor. For matrix, diagonal matrices, scalars, covariance intersection fusion, the accuracy of above four kinds of weighted fusion estimator is from high to low. But the computational burden is on the contrary. Covariance intersection fusion has minimal computational burden because of avoiding computing the cross covariance matrix. Therefore, it is suitable for real time applications from the viewpoint of engineering application.

(5) The proposed algorithm can be applied to robotics, economic, electronic, chemical systems and other fields.

\section{Acknowledgements}

This work is supported by Key Laboratory of Electronics Engineering, College of Heilongjiang Province, (Heilongjiang University), P. R. China, by science and technology research foundation of Heilongjiang education department under Grant 12531159, by the Natural Science Foundation of Heilongjiang Province of China (Grant Nos. F201426), by Harbin University of Commerce Youth Fund.

*Corresponding author: Gang Hao.

\section{References}

[1] K. Yang, Y. X. Shen and Z. C. Ji, "Robust $\mathrm{H}_{\infty}$ guaranteed cost control for uncertain switched singular systems with time-varying delay", Control Theory \& Applications, vol. 30, no. 3, (2013), pp. 787-790.

[2] X. H. Liu and X. Han, "Robust model predictive control for stochastic singular systems with polytopic uncertainty based on state observer", Control and Decision, vol. 28, no. 4, (2013), pp. 600-604.

[3] X. J. Sun and G. M. Yan, "Self-tuning weighted measurement fusion white noise deconvolution estimator and its convergence analysis", Digital Signal Processing, vol. 23, no. 1, (2013), pp. 38-48.

[4] P. J. Dua, S. C. Liu, J. S. Xia and Y. D. Zhao, "Information fusion techniques for change detection from multi-temporal remote sensing images", Information Fusion, vol. 14, no. 1 (2013), pp. 19-27.

[5] B. Khaleghi, A. Khamis, F. O. Karray and S. N. Razavi, "Multisensor data fusion: A review of the state-of-the-art", Information Fusion, vol. 14, no. 1, (2013), pp. 28-44.

[6] P. Cui, H. S. Zhang, H. X. Wang and J. E. Feng, "Globally optimal real-time distributed fusion of multi-channel observation systems", Control Theory \& Applications, vol. 8, no. 6, (2014), pp. $384-388$.

[7] M. B. Trawicki and M. T. Johnson, "Distributed multichannel speech enhancement with minimum mean-square error short-time spectral amplitude, log-spectral amplitude, and spectral phase estimation", Signal Processing, vol. 92, no. 2, (2012), pp. 345-356.

[8] G. Hao, X. F. Ye and T. Chen, "Weighted measurement fusion algorithm for nonlinear unscented Kalman filter”, Control Theory \& Applications, vol. 28, no. 6, (2011), pp. 753-758.

[9] J. X. Feng, Z. D. Wang and M. Zeng, "Distributed weighted robust Kalman filter fusion for uncertain systems with auto correlated and cross-correlated noises", Information Fusion, vol. 14, no. 1, (2013), pp. 78-86.

[10] Z. L. Deng, P. Zhang, W. J. Qi, Y. Gao and J. F. Liu, "The accuracy comparison of multisensor covariance intersection fuser and three weighting fusers", Information Sciences, vol. 14, no. 2, (2013), pp. 177-185.

[11] W. J. Qi, P. Zhang and Z. L. Deng, "Two-level robust sequential covariance intersection fusion Kalman predictors over clustering sensor networks with uncertain noise variances", International Journal of Sensor Networks, vol. 14, no. 4, (2013), pp. 251-261.

[12] W. J. Qi, P. Zhang and Z. L. Deng, "Covariance Intersection Fusion Kalman Estimators for Multi-Sensor System with Colored Measurement Noises", Research Journal of Applied Sciences, Engineering and Technology, vol. 6, no. 10, (2013), pp. 1872-1878.

[13] S. Y. Feng, W. W. Huang and C. G. Atkeson, "Decoupled state estimation for humanoids using full-body dynamics", Robotics and Automation (ICRA), (2014), pp. 195-201. 
[14] R. Xiong, F. C. Sun, Z. Chen and H. W. He, "A data-driven multi-scale extended Kalman filtering based parameter and state estimation approach of lithium-ion olymer battery in electric vehicles", vol. 113, (2014), pp. 463-476.

[15] S. L. Sun and Z. L. Deng, "Multi-sensor optimal information fusion Kalman filter", Automatica, vol. 40, no. 6, (2004), pp. 1017-1023.

[16] Y. X. Yuan and W.Y. Sun, "Optimization theory and methods", Beijing, Science Press (2003), pp. 69-74.

\section{Authors}

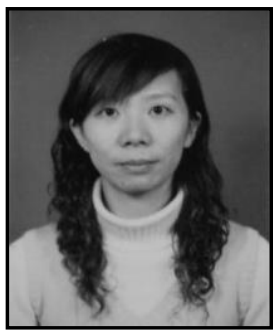

Yun Li, she is an associate professor at Harbin University of Commerce now. She obtained her bachelor's degree and master's degree in Heilongjiang University. Her major researches are state estimation, information fusion, etc.

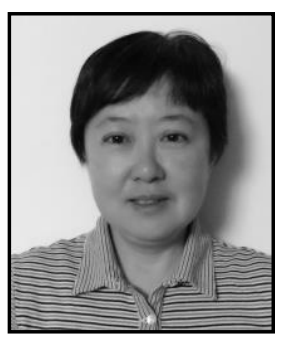

Ming Zhao, she is an associate professor at Harbin University of Commerce now. She obtained her bachelor's degree and master's degree in Harbin Engineering University. Her major researches are pattern recognition, information fusion, etc.

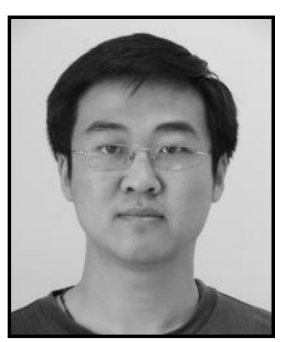

Gang Hao, he is an associate professor at Heilongjiang University now. He obtained his bachelor's degree and master's degree in Heilongjiang University, and obtained his Ph.D. in Harbin Engineering University. His major researches are state estimation, information fusion, etc. 
International Journal of Control and Automation Vol. 8, No.4 (2015) 